\title{
Development of a Five-Degree-of-Freedom Seated Human Model and Parametric Studies for Its Vibrational Characteristics
}

\author{
Jong-Jin Bae and Namcheol Kang (iD \\ School of Mechanical Engineering, Kyungpook National University, Daegu, Republic of Korea \\ Correspondence should be addressed to Namcheol Kang; nckang@knu.ac.kr
}

Received 28 June 2018; Revised 22 September 2018; Accepted 30 September 2018; Published 23 October 2018

Academic Editor: Giuseppe Ruta

Copyright ( 92018 Jong-Jin Bae and Namcheol Kang. This is an open access article distributed under the Creative Commons Attribution License, which permits unrestricted use, distribution, and reproduction in any medium, provided the original work is properly cited.

\begin{abstract}
This study focuses on the biodynamic responses of a seated human model to whole-body vibrations in a vehicle. Five-degree-offreedom nonlinear equations of motion for a human model were derived, and human parameters such as spring constants and damping coefficients were extracted using a three-step optimization processes that applied the experimental data to the mathematical human model. The natural frequencies and mode shapes of the linearized model were also calculated. In order to examine the effects of the human parameters, parametric studies involving initial segment angles and stiffness values were performed. Interestingly, mode veering was observed between the fourth and fifth human modes when combining two different spring stiffness values. Finally, through the frequency responses of the human model, nonlinear characteristics such as frequency shift and jump phenomena were clearly observed.
\end{abstract}

\section{Introduction}

Vibrational characteristics of seated humans are an important consideration in the automotive industry because they play a major role in riding comfort. Furthermore, recent significant advances in electric and autonomous vehicles may affect the perception and emotions of occupants in a vehicle. For example, in conventional vehicles, combustion engine-induced vibration and noise act in concert to mask road-induced vibration and noise; generally, this does not occur with electric vehicles. Thus, we can expect that drivers and passengers will be more sensitive to road-induced vibrations, and that the importance of analyzing vibrational perception in vehicles will increase.

The characteristics of the dynamic responses of a seated human body are mainly affected by low-frequency vibrations (below $50 \mathrm{~Hz}$ ). A number of studies have also found that the fundamental frequency of a seated human exposed to wholebody vibration is lower than $10 \mathrm{~Hz}[1-5]$. The natural frequencies and frequency responses of a seated human are decided depending on body weight, length of human segments, and sitting posture (e. g., slouched or erect) [6-10]. In addition, dynamic characteristics of a seated human involve nonlinearities such as the frequency softening phenomenon, in which the first natural frequency decreases as the magnitude of the excited vibrations increases [11-13]. Mansfield and Griffin [11] inferred that complex human factors such as muscle forces and the bending or buckling of a spine could give rise to these nonlinearities. Therefore, it is challenging to quantitatively analyze the dynamic responses of a seated human body, owing to its complexity and nonlinearity.

Various finite element models of the human have been designed to investigate the complex characteristics of a seated human. Vavalle et al. [14] introduced the whole body finite element model, for conducting dynamic simulations under impact loading conditions. Siefert et al. [15] developed a human-seat interaction system and extracted the seat transfer functions and contact pressure between a human body and a seat. Butz et al. [16] proposed the finite element model of the lower extremity based on anatomy data and calculated the dynamic responses of the human model in the military vehicles. Although the finite element method is capable of sophisticated modelling of the entire human body and local segments, its computational time increases as the number of elements increases in nature. In addition, it is not straightforward to develop finite element models of a human body that can accommodate variations in geometric parameters such as body segment lengths and sitting postures. 
To overcome the disadvantages of finite element models, lumped parameter models consisting of masses, springs, and dampers have been widely emphasized in various studies. Wei and Griffin [17] utilized single-degree- and two-degree-offreedom human models to mathematically predict seat transmissibility. They also revealed that the two-degree-offreedom provided the better fitness to the experimental results than the single-degree-of-freedom. Choi and Han [18] used the vertical seat-human model that calculated the vibrational performance of semiactive seat suspensions quantitatively with SEAT values and vibration dose value (VDV). Bai et al. [19] analyzed the dynamic characteristics of a four-degree-offreedom biodynamic model with various structural configurations to identify the best configuration. An improved generic algorithm was considered to determine the human parameters. Although these human models that take only translational motions into account can be used to describe frequency responses of occupants exposed to whole-body vibrations, they cannot represent the swing motions of human segments, owing to the absence of rotational degrees-of-freedom. Therefore, in order to more accurately grasp and analyze the dynamic movements of human segments, it is essential to develop a biodynamic human model that considers rotational motions.

Lumped human models with rotational degrees-of-freedom have been studied extensively to interpret fore-and-aft and pitch movements in the sagittal plane. Matsumoto and Griffin [20] developed four- and five-degree-of-freedom human models including the viscera with rotational springs and dampers, and they showed that the models having the rotational degree-of-freedom represented the more reasonable descriptions for the dynamic characteristics of the seated human body. Cho and Yoon [21] presented nine-degree-offreedom human model consisting of three rigid bodies and compared with the single-, two-, and three-degree-of-freedom models to verify the performance of the proposed ninedegree-of-freedom human model. Further, they also investigated the effect of the seat backrest. Kim et al. [22] proposed a seven-degree-of-freedom seat-mannequin model whose parameters were obtained using indentation and swing experimental tests; the frequency response functions of the seat-mannequin model were presented and compared to the experimental results. Zheng et al. [23] proposed sevendegree-of-freedom human model and showed analytical and experimental apparent masses with and without a backrest in the vertical and fore-and-aft directions. Also, the sensitive parameters were presented according to the presence or absence of backrest. Mohajer et al. [24] presented a human biomechanical model that consisted of 15 rigid bodies. The ride comfort was also estimated with respect to road roughness, vehicle speed, and the weights of the subjects using the proposed human model.

To develop the human model, we used a Lagrangian formulation to derive the nonlinear equations of motion for a five-degree-of-freedom model. The spring constants and damping coefficients were extracted from experimental data in the literature using an optimization process. The natural frequencies and mode shapes were also calculated from the linearized human model. In addition, several parametric studies were performed. Finally, we calculated the frequency response curves of the nonlinear human model, and then compared those of the linear model, nonlinear model, and the experiment.

\section{Nonlinear and Linear Five-Degree-of-Freedom Models}

2.1. Model Description. In order to investigate dynamic characteristics of a seated human, we developed a lumped parameter model consisting of masses, dampers, and springs. In the proposed model, we considered three rigid bodies- a head, trunk, and lower body (including the thighs and pelvis). The trunk and lower body are the suggested measurement points of vibrations in BS 6841, which is the standard for assessing human vibrations [25]. Further, the motions of the head-neck segment are a prominent source of discomfort [10] because they may affect the vision of vehicle occupants and may cause motion sickness. Foot support was not considered because the vibrations transmitted through foot supports are relatively small compared to the vibrations affecting the trunk and lower body [26].

Based on these assumptions, we developed a five-degreeof-freedom human model consisting of $x_{\mathrm{h}}, z_{\mathrm{h}}, \theta_{1}, \theta_{2}$, and $\theta_{3}$, as shown in Figure $1 x_{\mathrm{h}}$ and $z_{\mathrm{h}}$, respectively, represent the horizontal and vertical displacements of the hip joint with respect to the basi-centric coordinate system, while $\theta_{1}, \theta_{2}$, and $\theta_{3}$, respectively, represent the angular displacements of the lower body, trunk, and head around the $y$-axis. Because we focus our interpretation on analyzing motions in the rotational and vertical directions at the $x-z$ plane (sagittal plane), dynamic behavior in the lateral direction was not considered. The base motion $z_{\mathrm{b}}$ is the input vertical displacement and velocity of the seat floor. It was assumed that no components overlapped, and each segment was connected to a torsional spring and damper to represent the rotational motion. The translational springs were connected to the surface of each component. It is also assumed that each human segment has uniform mass distribution. The joints of the human model were modelled using the pin joint condition because the elongation and compression at the rotational joints of an actual human body are very small. In other words, the independent translational movements of each segment were not considered in our model.

The combination of seat foam and human skin was considered as the translational springs and dampers. All springs assumed to be massless and frictionless were in nonstretched and nonrotated conditions at the initial configuration. Although seat foam has nonlinear viscoelastic properties [27-29], it has been reported that such properties do not significantly affect the transmissibility of human body vibrations in practice [13]. It was also assumed that because deformations of the human back and buttock tissue after sitting on a seat are sufficiently small, their nonlinearity could be disregarded. Therefore, considering the combination of seat foam and human tissue as the linear translational spring and damper provides a convenient and reasonable approach to developing this lumped parameter human model. The backrest was set to be parallel to the trunk of the human model at the initial configuration. 


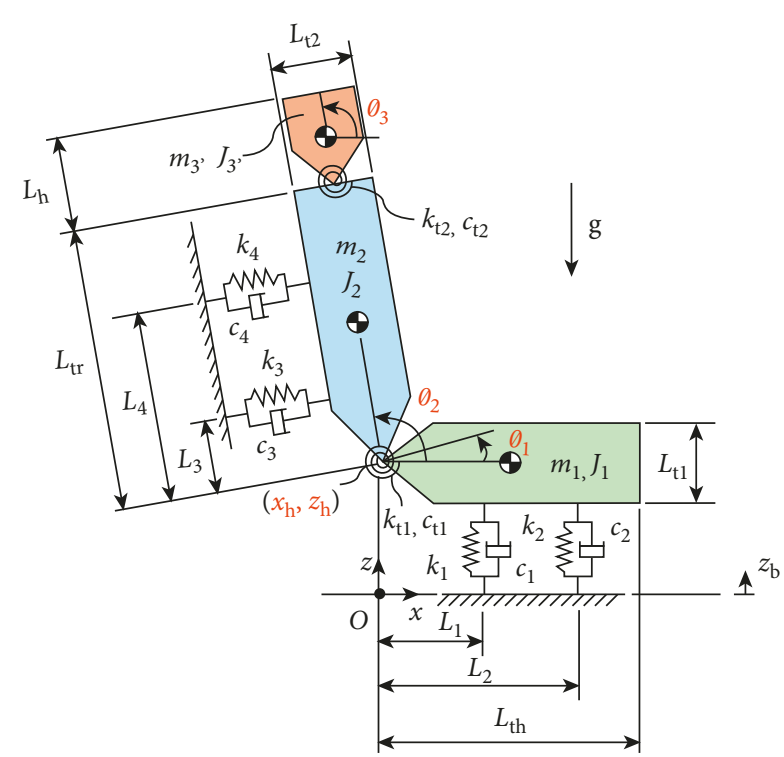

FIGURE 1: The proposed five-degree-of-freedom seated biodynamic human mathematical model (red colored symbols: generalized coordinates).

It is necessary to define translational spring deformation values to reflect actual deformation characteristics of the seat foam and tissue. Unlike vertical human models comprising one-dimensional motion in vertical directions, each segment of the proposed five-degree-of-freedom model is able to represent horizontal and rotational motions. When the deformation value of the translational spring is defined by the distance between two connected points, the spring forces are generated in the diagonal direction under this definition. In the case of actual foam and tissue deformation, when the contact points are moved, the foam of the seat located at the moved position generates the force to support a human body in the normal direction. To this end, the tangential force of the translational spring was disregarded, and the deformation of the translational spring in the normal direction of the seat floor and seatback was used for calculating the dynamic responses (Figure 2).

2.2. Derivation of Nonlinear Equations of Motion. The nonlinear equations of motion for the five-degree-of-freedom mathematical model were derived using the Lagrange equation. The locations of the center of mass at each segment are

$$
\begin{aligned}
& x_{1}=x_{\mathrm{h}}+\frac{1}{2} L_{\mathrm{th}} \cos \theta_{1}, \\
& x_{2}=x_{\mathrm{h}}+\frac{1}{2} L_{\mathrm{tr}} \cos \theta_{2}, \\
& x_{3}=x_{\mathrm{h}}+L_{\mathrm{tr}} \cos \theta_{2}+\frac{1}{2} L_{\mathrm{h}} \cos \theta_{3}, \\
& z_{1}=z_{\mathrm{h}}+\frac{1}{2} L_{\mathrm{th}} \sin \theta_{1}, \\
& z_{2}=z_{\mathrm{h}}+\frac{1}{2} L_{\mathrm{tr}} \sin \theta_{2}, \\
& z_{3}=z_{\mathrm{h}}+L_{\mathrm{tr}} \sin \theta_{2}+\frac{1}{2} L_{\mathrm{h}} \sin \theta_{3},
\end{aligned}
$$

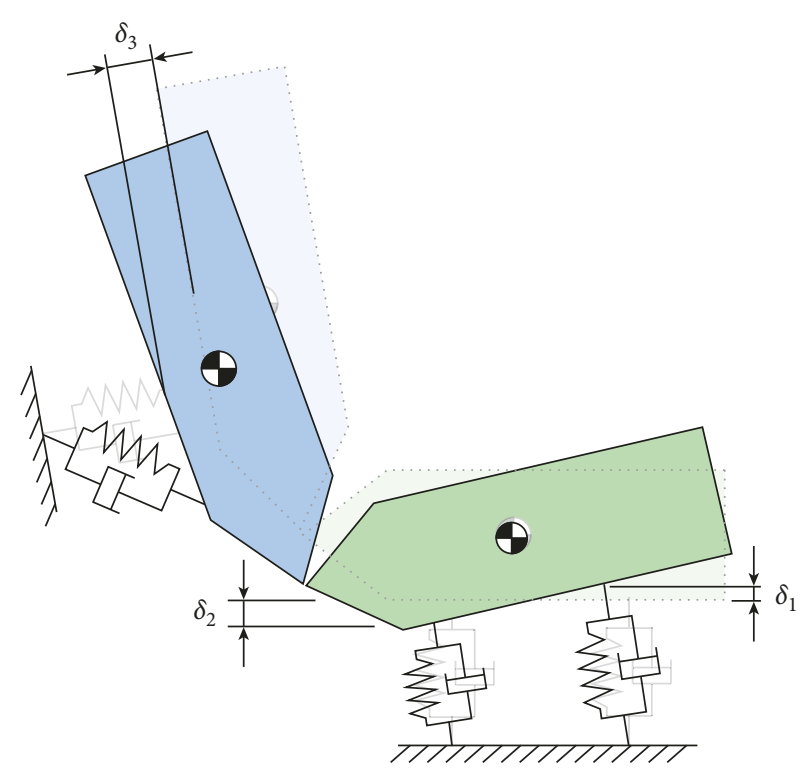

Figure 2: Directional definition of the translational spring deflections.

where $x_{\mathrm{h}}$ and $z_{\mathrm{h}}$ are the horizontal and vertical displacements of the hip joint, $x_{i}$ and $z_{i}$ are the locations of the centers of mass of the lower body, trunk, and head, respectively, and $L_{\mathrm{th}}, L_{\mathrm{tr}}$, and $L_{\mathrm{h}}$ define the lengths of the lower body, trunk, and head, respectively. The kinetic energy of the human model is derived as follows:

$$
T=\frac{1}{2} \sum_{i=1}^{3} m_{i}\left(\dot{x}_{i}^{2}+\dot{z}_{i}^{2}\right)+\frac{1}{2} \sum_{i=1}^{3} J_{i} \dot{\theta}_{i}^{2},
$$

where $m_{i}$ and $J_{i}$, respectively, indicate the mass and mass moment of inertia of the corresponding segment. It can be easily seen that translational and rotational kinetic energy are considered for three rigid bodies. The derived potential energy and Rayleigh's dissipation function are written as

$$
\begin{aligned}
& V=\frac{1}{2} \sum_{i=1}^{4} k_{i} \delta_{i}^{2}+\frac{1}{2} \sum_{i=1}^{2} k_{\mathrm{ti}} \delta_{\mathrm{ti}}^{2}+\sum_{i=1}^{3} m_{i} g \Delta_{i}, \\
& D=\frac{1}{2} \sum_{i=1}^{4} c_{i} \dot{\delta}_{i}^{2}+\frac{1}{2} \sum_{i=1}^{2} c_{\mathrm{t} i} \dot{\delta}_{\mathrm{ti}}^{2},
\end{aligned}
$$

where $V$ is the potential energy associated with the translational and torsional spring forces and gravity, $D$ is the Rayleigh's dissipation function representing linear functions of velocities, and $k_{i}, c_{i}$, and $g$, respectively, denote translational spring constants, damping coefficients, and gravitational acceleration. $k_{\mathrm{t} i}$ and $c_{\mathrm{t} i}$ denote torsional stiffness values and damping coefficients, respectively. $\delta_{i}$ and $\delta_{t i}$ are the displacements of the translational springs and torsional springs, respectively. Now, applying the Lagrange equation to Equations (1)-(4) yields 


$$
\left[\begin{array}{ccccc}
A_{11} & 0 & A_{13} & A_{14} & A_{15} \\
& A_{22} & A_{23} & A_{24} & A_{25} \\
& & A_{33} & 0 & 0 \\
& & & A_{44} & A_{45} \\
\text { Sym. } & & & & A_{55}
\end{array}\right]\left\{\begin{array}{l}
\ddot{x}_{h} \\
\ddot{z}_{h} \\
\ddot{\theta}_{1} \\
\ddot{\theta}_{2} \\
\ddot{\theta}_{3}
\end{array}\right\}=\left\{\begin{array}{c}
B_{1} \\
B_{2} \\
B_{3} \\
B_{4} \\
B_{5}
\end{array}\right\} .
$$

The elements of each matrix are given in Appendix A.

Prior to examining the dynamic characteristics using nonlinear equations of motion, linearization was performed. It is not a simple task to simulate the nonlinear human model to obtain dynamic characteristics such as the natural frequency, mode shape, and parametric sensitivity, and excessive computational time would be required. Therefore, we carried out the linearization by expending the Taylor series at the initial configurations, which makes it possible to represent the equations of motion with a set of generalized coordinates. In the linearization of nonlinear equations of motion, the following approximations were considered:

$$
\begin{aligned}
& \sin \theta=\sin \left(\theta^{*}+\theta_{0}\right) \approx \sin \theta_{0}+\cos \theta_{0} \cdot \theta^{*}, \\
& \cos \theta=\cos \left(\theta^{*}+\theta_{0}\right) \approx \cos \theta_{0}-\sin \theta_{0} \cdot \theta^{*},
\end{aligned}
$$

where $\theta^{*}$ denotes a small perturbation around the initial configuration. The linearized equations of motion can be written as

$$
\mathbf{M} \ddot{\mathbf{x}}+\mathbf{C} \dot{\mathbf{x}}+\mathbf{K x}=\dot{z}_{\mathrm{b}} \mathbf{C}_{\mathrm{b}}+z_{\mathrm{b}} \mathbf{K}_{\mathrm{b}},
$$

where $\mathbf{x}^{\mathrm{T}}$ is the generalized coordinate vector $\left(x_{\mathrm{h}}^{*}, z_{\mathrm{h}}^{*}, \theta_{1}^{*}, \theta_{2}^{*}, \theta_{3}^{*}\right)^{\mathrm{T}}$, and each matrix and vector is configured as follows:

$$
\begin{aligned}
& \mathbf{M}=\left[\begin{array}{ccccc}
M_{11} & 0 & M_{13} & M_{14} & M_{15} \\
& M_{22} & M_{23} & M_{24} & M_{25} \\
& & M_{33} & 0 & 0 \\
& & & M_{44} & M_{45} \\
\text { Sym. } & & & & M_{55}
\end{array}\right], \\
& \mathbf{C}=\left[\begin{array}{ccccc}
C_{11} & C_{12} & 0 & C_{14} & 0 \\
& C_{22} & C_{23} & C_{24} & 0 \\
& & C_{33} & C_{34} & 0 \\
& & & C_{44} & C_{45} \\
\text { Sym. } & & & & C_{55}
\end{array}\right], \\
& \mathbf{K}=\left[\begin{array}{ccccc}
K_{11} & K_{12} & 0 & K_{14} & 0 \\
& K_{22} & K_{23} & K_{24} & 0 \\
& & K_{33} & K_{34} & 0 \\
& & & K_{44} & K_{45} \\
\text { Sym. } & & & & K_{55}
\end{array}\right] \\
& \mathbf{C}_{\mathrm{b}}=\left[\begin{array}{lllll}
0 & C_{\mathrm{b} 2} & C_{\mathrm{b} 3} & 0 & 0
\end{array}\right]^{\mathrm{T}}, \\
& \mathbf{K}_{\mathrm{b}}=\left[\begin{array}{lllll}
0 & K_{\mathrm{b} 2} & K_{\mathrm{b} 2} & 0 & 0
\end{array}\right]^{\mathrm{T}} \text {. }
\end{aligned}
$$

The elements of each matrix are given in Appendix B. Taking the Fourier transform of Equation (7), the linear equations of motion in the frequency domain are given by

$$
\mathbf{X}(j \omega)=\left(-\omega^{2} \mathbf{M}+j \omega \mathbf{C}+\mathbf{K}\right)^{-1}\left(j \omega \mathbf{C}_{\mathrm{b}}+\mathbf{K}_{\mathrm{b}}\right) Z_{\mathrm{b}}(j \omega)
$$

Thus, we can calculate the natural frequency and mode shape of the human model using the mass and stiffness matrix. The seat-to-head (STH) transmissibility of the linear model can be computed from Equation (9); it will be used to determine the human parameters by a comparison with the experimental results.

2.3. Identification of Human Parameters. The inertial and geometric properties, stiffness, and damping coefficients are crucial parameters that determine the dynamic responses of the proposed human model. In this study, we determined the human parameters through the following three steps:

(1) Determination of inertial and geometric properties

(2) Extraction of stiffness values

(3) Extraction of damping coefficients.

The inertial and geometric parameters were measurable using the measurement tools; further, we chose the mass, moment of inertia, and human segment length data from the reported literature related to anthropometry. By contrast, the stiffness and damping coefficients are not easy to measure experimentally, and thus we extracted these parameters by using the optimization process from the experimental STH transmissibility results [30]. In the second and third steps, we determined the stiffness values and damping coefficients.

2.3.1. Determination of Inertial and Geometric Parameters. In the initial step, we determined the mass, moment of inertia, and segment length values. In the second and third step to obtain the stiffness and damping coefficients, the experimental subjects in the whole-body experiments were Korean males in their late 20s [21, 30], and thus we tried to select the mass and geometric parameters from Korean anthropometric data as close as possible to these experimental subjects [31, 32]. However, we used the mass moment of inertia from alternative anthropometric data, which is the similar configuration of the proposed human model, because of a lack of validated official data for Korean males [22]. The human mathematical model in this study had a backrest angle of $111^{\circ}$, a seat pan angle of $12^{\circ}$ [12], and a head angle of $100^{\circ}$ (to the horizontal line) [22]. The mass, mass moment of inertia, and geometric length values used in the human mathematical model are listed in Table 1.

\subsubsection{Extraction of the Stiffness and Damping Coefficients.} In this study, the springs and dampers underneath the lower body and the springs and dampers connected between the backrest and trunk were set to have different stiffness values and damping coefficients. The foam of the seatback shows the different force-displacement relationship from the seat pad [33]. However, in the sitting condition on a seat, it is assumed that the stiffness of a human body in the vicinity of the buttock and thigh has approximately identical value in the case of small penetration based on loaddeflection measurement [34]. Furthermore, the deformation 
TABLE 1: Inertial properties and length data of the five-degree-offreedom human model.

\begin{tabular}{lcc}
\hline & Property & Value \\
\hline \multirow{2}{*}{ Mass $(\mathrm{kg})$} & $m_{1}$ & 10.49 \\
& $m_{2}$ & 33.98 \\
& $m_{3}$ & 6.67 \\
\hline \multirow{3}{*}{ Mass moment of inertia $\left(\mathrm{kg} \cdot \mathrm{m}^{2}\right)$} & $J_{1}$ & 0.23 \\
& $J_{2}$ & 2.05 \\
& $J_{3}$ & 0.03 \\
\hline & $L_{\mathrm{tr}}$ & 598.60 \\
& $L_{\mathrm{th}}$ & 571.70 \\
& $L_{\mathrm{h}}$ & 217.10 \\
& $L_{1}$ & 88.00 \\
& $L_{2}$ & 459.80 \\
& $L_{3}$ & 100.00 \\
& $L_{4}$ & 478.90 \\
& $L_{\mathrm{t} 1}$ & 156.20 \\
& $L_{\mathrm{t} 2}$ & 224.00 \\
\hline
\end{tabular}

characteristics of the foam in the seatback or seat pad were assumed to be similar; therefore, we assumed that the stiffness and damping coefficients of the translational springs and dampers connected to the same component of the human model have the equal values, respectively (i.e., $k_{1}$ $=k_{2}, k_{3}=k_{4}, c_{1}=c_{2}$, and $c_{3}=c_{4}$ ).

To identify the stiffness and damping coefficients, the experimental STH transmissibility was obtained from the reported literature [30]. In the optimization to obtain human parameters, we used the "fmincon" function in MATLAB (2015b), which is known to be effective for convex optimization. Firstly, the stiffness values were extracted based on the natural frequency of the experimental results. Because the estimation of the natural frequency tends to be more repeatable than the estimation of amplitude in general vibration experiments, we considered that the natural frequency values reported in the literature are more reliable than those for amplitude; we subsequently prioritized determining the natural frequency of the human model. We then determined the damping coefficients by minimizing the difference between the amplitude of the human mathematical model and the experiment.

The stiffness of the five-degree-of-freedom human model was determined based on the first and second natural frequencies $(4.2 \mathrm{~Hz}$ and $7.5 \mathrm{~Hz})$ from the experimental results. The following objective function was used in the process for determining the stiffness values:

$$
E_{1}=\min \left(w_{1}^{(2)}\left|f_{1}^{\mathrm{ex}}-f_{1}^{\mathrm{cal}}\right|+w_{2}^{(2)}\left|f_{2}^{\mathrm{ex}}-f_{2}^{\mathrm{cal}}\right|\right),
$$

where $f_{1}^{\text {ex }}, f_{2}^{\text {ex }}, f_{1}^{\text {cal }}, f_{2}^{\text {cal }}$ denote the first and second natural frequency of the experiment and the proposed human model, respectively. $w_{1}^{(2)}, w_{1}^{(2)}$ are the weighting factors (2.0 and 1.0 were applied, respectively). The superscripts indicate the second step. We calculated the natural frequencies of the human model using a mass and stiffness matrix whose elements are optimized spring constants, and both results indicate the same natural frequency, as listed in Table 2 . The mode shape was also computed, as shown in Figure 3; it will be explained in detail in Section 3.2.
TABle 2: Natural frequencies of the proposed human model and experiment.

\begin{tabular}{lcc}
\hline \multirow{2}{*}{ Mode } & \multicolumn{2}{c}{ Natural frequency $(\mathrm{Hz})$} \\
& Proposed model & Experiment \\
\hline 1 & 4.20 & 4.20 \\
2 & 7.50 & 7.50 \\
3 & 9.99 & - \\
4 & 19.70 & - \\
5 & 21.35 & - \\
\hline
\end{tabular}

The objective function for the extraction of the damping coefficient is as follows:

$$
E_{2}=\min \left(w_{1}^{(3)} D_{1}+w_{2}^{(3)} \mathrm{D}_{2}\right)
$$

where

$$
\begin{aligned}
& D_{1}=\frac{\int_{f_{\mathrm{a}}}^{f_{\mathrm{b}}}\left|T^{\mathrm{ex}}\left(f_{i}\right)\right|-\left|T^{\mathrm{cak}}\left(f_{i}\right)\right| d f}{\int_{f_{\mathrm{a}}}^{f_{\mathrm{b}}}\left|T^{\mathrm{ex}}\left(f_{i}\right)\right| d f}, \\
& D_{2}=\left|\max \left(T^{\mathrm{ex}}\right)-\max \left(T^{\mathrm{cal}}\right)\right|,
\end{aligned}
$$

where $T^{\mathrm{ex}}$ is the experimental STH transmissibility and $T^{\mathrm{cal}}$ is the STH transmissibility of the human mathematical model. $f_{\mathrm{a}}$ and $f_{\mathrm{b}}$ are the first and final frequencies of the experimental data $(0 \mathrm{~Hz}$ and $20 \mathrm{~Hz}$, respectively). In order to maximize the correlation between our calculated amplitudes and the experimental results near the first natural frequency, the weighting factors $w_{1}^{(3)}, w_{1}^{(3)}$ are set to 1.0 and 2.0 , respectively. The initial and optimized stiffness and damping coefficients are listed in Table 3.

It can be seen that the first natural frequency and the maximum amplitude of the STH transmissibility of the human mathematical model calculated from the optimized parameters are consistent with those of the experimental STH transmissibility, as shown in Figure 4. The frequency response curve of the human model around the fundamental frequency is in good agreement with that of the experimental STH transmissibility in the range of $0-7 \mathrm{~Hz}$. However, both results differ at frequencies ranging from $7-15 \mathrm{~Hz}$. In practice, it is not easy to simultaneously represent the first and second peaks in the frequency response curves using our model, owing to the limited configurations of the proposed human model. Thus, we focused on fitting to the amplitude of the first natural frequency in the frequency domain. Typically, in the frequency response curves of a seated human, the first resonance frequency is clearly distinguished from the higher mode. Moreover, similar frequency bands and amplitudes are reported in many studies $[1,6,15,21]$. However, the second natural frequency of a seated human is relatively undetectable and is significantly affected by individual subject characteristics such as sitting posture, body weight, and length of human segments. For example, Rakheja et al. [6] reported the apparent masses of seated subjects with respect to their sitting postures. In this literature, the clarity of the second peak is dependent on the body mass, measurement point, and magnitude of the excited vibrations. In addition, the amplitude of the fundamental 

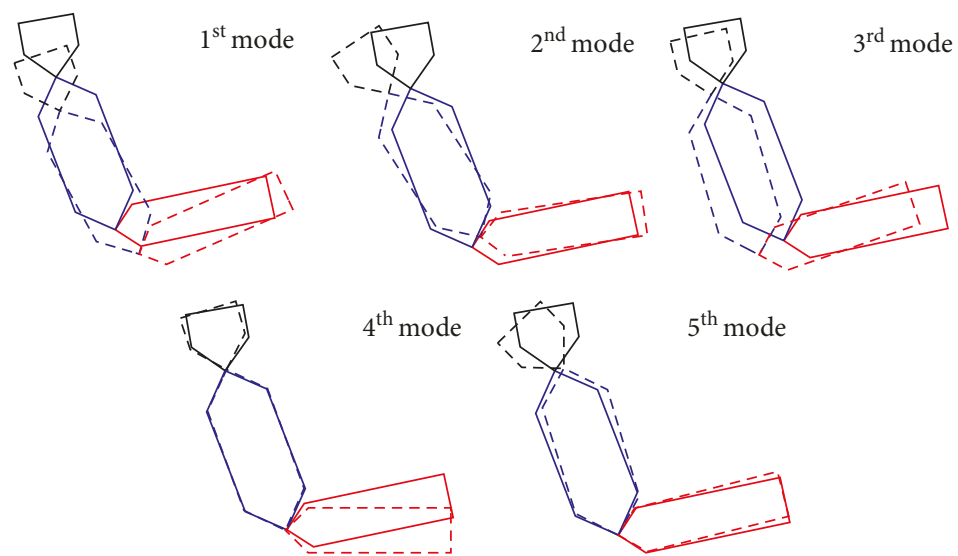

Figure 3: Mode shapes of the five-degree-of-freedom human model (solid line: initial configuration; dashed line: mode shape configuration; black color: head; blue color: trunk; red color: lower body).

TABLE 3: Initial and optimized values of the stiffness and damping coefficients.

\begin{tabular}{lccc}
\hline Parameter & Parameter & Initial value & Optimized value \\
\hline & $k_{1}, k_{2}$ & 80.00 & 66.60 \\
Stiffness & $k_{3}, k_{4}$ & 100.00 & 95.54 \\
$(\mathrm{kN} / \mathrm{m}, \mathrm{kNm} / \mathrm{rad})$ & $k_{\mathrm{t} 1}$ & 2.00 & 1.42 \\
& $k_{\mathrm{t} 2}$ & 1.00 & 1.12 \\
\hline & $c_{1}, c_{2}$ & 1.50 & 0.89 \\
Damping coefficient & $c_{3}, c_{4}$ & 1.50 & 0.94 \\
$(\mathrm{kNs} / \mathrm{m}, \mathrm{kNms} / \mathrm{rad})$ & $c_{\mathrm{t} 1}$ & 0.30 & 0.30 \\
& $c_{\mathrm{t} 2}$ & 0.20 & 0.20 \\
\hline
\end{tabular}

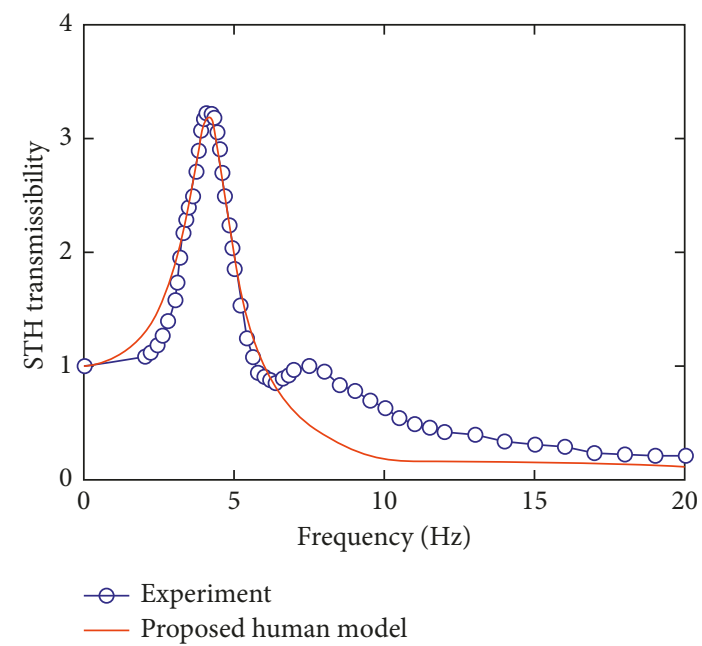

FIGURE 4: STH transmissibility of experimental results [30] and the five-degree-of-freedom human linear model calculated using the optimized stiffness and damping coefficients.

frequency is much greater than that of the higher-order natural frequency, and it can be inferred that fitting to the first natural frequency represents the dynamic characteristics more accurately. In this sense, it can be concluded that the optimized parameters of the proposed human model can adequately represent the dynamic characteristics of the human body.

\section{Study of Parameters in Human Linear Model}

Understanding how parameters affect the dynamic characteristics of the human model is an important issue in the mechanical approach to explaining the dynamic responses of the human body. Such understanding also requires parametric analysis of the human mathematical model, which is achieved by varying the human parameters. Thus, we analyzed the variations in the natural frequencies of the human model according to changes in the parameters, which mainly affect the dynamic responses of the proposed model. In order to conduct the parameter study, we selected the inclination angles of the human model, including seat pan, backrest and head angles, and the translational and torsional stiffness values.

3.1. Variations in Rotational Angles. Parameter studies focused on the angle of the seat pan, backrest, and head were performed in order to analyze the variations in natural frequencies caused by varying the sitting posture. In the linear human model, the angles of the seat pan, backrest, and head were defined as $\theta_{10}, \theta_{20}$, and $\theta_{30}$, respectively. The optimized stiffness and damping coefficients were applied to the parametric study, and the natural frequencies of the human mathematical model were calculated when $\theta_{10}, \theta_{20}$, and $\theta_{30}$ were changed in turn.

Figure 5 shows the variations in natural frequency that resulted from variations in the inclination angles. In this figure, the first, second, and third columns, respectively, indicate the resonance frequency with respect to the seat pan, backrest, and head angle, and the first to fifth rows represent the order of the natural frequency. The $y$-axis scale with respect to the inclination angle was determined based on the sensitivity of the corresponding parameter.

The changes in natural frequencies with respect to the seat pan angle are shown in the first column of Figure 5. The angle of the seat pan was changed in the range of $0^{\circ}$ to $20^{\circ}$ at $2^{\circ}$ intervals. We set the $y$-axis scale to $\pm 5 \%$ of the corresponding natural frequency because the variation in all natural frequencies is relatively small. The black dotted line indicates the baseline value of the seat pan angle. 

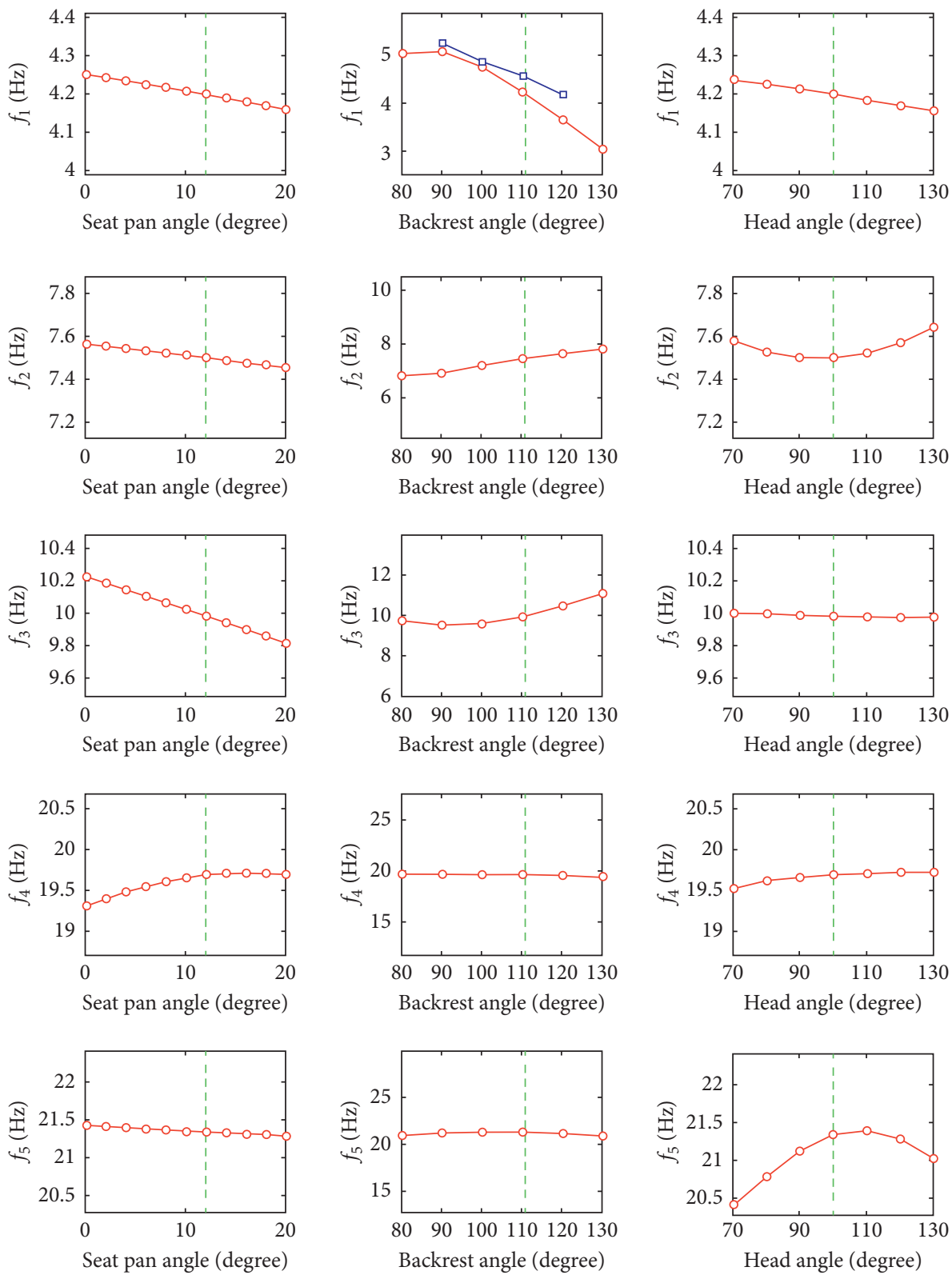

Figure 5: Natural frequencies of the five-degree-of-freedom linear model with respect to the seat pan, backrest, and head angle (dotted vertical green line: baseline angle; blue line in the graph in the second column of the first row: experimental results [35]; the top through the bottom rows represent the first through the fifth modes, respectively).

As can be seen, the natural frequencies of the human mathematical model are not significantly affected by the change in the seat pan angle. As the seat pan angle increases, the natural frequencies excluding the fourth mode tend to decrease as a whole, but the variation values are less than $5 \%$. These tendencies were also observed in the experimental results. Wang et al. reported no statistical significance between the variations in the seat pan angle and the variations in the first natural frequency of the human body [8].

The second column of Figure 5 shows the variations in natural frequencies caused by varying the backrest angle from $80^{\circ}$ to $130^{\circ}$ at $10^{\circ}$ intervals. The $y$-axis scale was set to $\pm 30 \%$ of the corresponding order of the natural frequency. To verify the human mathematical model, we performed a comparison between the first natural frequencies of the human model and the experiment. The experimental natural frequency resulting from varying the backrest angle is shown in Figure 5 in the second column of the first row as the solid blue line; in this case, the foam thickness is $150 \mathrm{~mm}$ [35]. The fundamental frequency of the human model was reduced by approximately $20 \%$ as the backrest angle increased from $80^{\circ}$ to $130^{\circ}$, and the experimental results show a similar tendency. From this tendency, it is inferred that the human back muscles become relaxed as the backrest angle increases, and that the first natural frequency decreases owing to the reduction in the stiffness of the human back [35]. In addition, the second natural frequency increases as the backrest angle increases, and 
the third natural frequency slightly decreases and then increases. There was no significant change in the fourth and fifth natural frequencies according to the backrest angle change. Therefore, it can be expected that our human mathematical model enables us to represent variations in the first natural frequency through comparisons with experimental data.

The variations in the natural frequencies with respect to the head angle were also calculated, as shown in the third column of Figure 5. The head angles were varied between $70^{\circ}$ and $130^{\circ}$ at intervals of $10^{\circ}$. The $y$-axis scale was set to $\pm 5 \%$ of the corresponding natural frequency. As the head angle increased, all natural frequencies had different tendencies but did not change substantially. Similar to the results of the change in the seat pan angle, the head angle seems to have only a minor role in the natural frequencies of the human model.

3.2. Effect of Translational and Torsional Stiffness. To investigate the effect of the translational and torsional spring constants, we analyzed the change in the natural frequencies according to the change in stiffness (Figure 6). The natural frequency of the human model was calculated by changing the baseline stiffness values from 1/1000 to two times the baseline value. The black dotted lines indicate the baseline stiffness values.

The first natural frequency increases with an increase in $k_{1}$, and the change in $k_{2}$ does not have a significant effect. Also, the first natural frequency slightly increases with an increase in $k_{3}$ and $k_{4}$ when $k_{3}$ and $k_{4}$ are greater than the baseline. The variations in $k_{\mathrm{t} 1}$ and $k_{\mathrm{t} 2}$, which, respectively, represent the hip joint and head/neck joint, do not significantly affect the first natural frequency. These tendencies can also be confirmed from the configurations of the corresponding mode shape. It can be expected that the variations in the $k_{2}$ stiffness spring, which is connected relatively far from the hip joint, would not have a significant effect on the first mode because the first mode shape is comprised mainly of the vertical motion of the hip joint. In addition, the rotational motions of the head and trunk are relatively small compared to the vertical motion of the hip joint; thus, the torsional spring has a very small effect on variations in the first natural frequency. In summary, the fundamental frequency of the human model was sensitive to the changes in $k_{1}, k_{3}$, and $k_{4}$ stiffness; in particular, the variations in $k_{1}$ stiffness play a major role in the first mode.

The second natural frequency was sensitive to $k_{3}, k_{4}, k_{\mathrm{t} 1}$, and $k_{\mathrm{t} 2}$ stiffness. In particular, the effects of varying $k_{3}$ and $k_{4}$ are more significant. The change in $k_{3}$ stiffness has a minor effect when the $k_{4}$ value varies below the baseline value, but the second natural frequency is varied considerably owing to an increase in the $k_{3}$ value when the $k_{4}$ value is greater than the baseline. Moreover, it can be seen that the second natural frequency indicates a minor change with respect to the variations in $k_{1}$. The dominant motion of the second mode is the rotation of the trunk. Thus, the translational motion of the hip joint and the rotation of the head and thigh are not significant. Therefore, the springs that affect the rotation of the trunk are $k_{3}, k_{4}$, and $k_{\mathrm{t} 1}$, owing to its mode shape. The third mode is particularly influenced by the changes in the translational stiffness values $k_{3}$ and $k_{4}$. The stiffness values of the $k_{3}$ and $k_{4}$ springs, which connect the backrest and trunk, play an important role because the hip joint moves in the horizontal direction. The other stiffness values also have relatively little influence on the third-order natural frequency. It can be seen that $k_{\mathrm{t} 2}$ and $k_{2}$ are the most influential parameters in the fourth and fifth modes. The configurations of corresponding mode shapes support these findings, and the rotational motions of the head and lower body occur simultaneously in the fourth and fifth modes. These modes have no impact on the variations in the hip joint torsional spring $k_{\mathrm{t} 1}$ or the translational springs $k_{3}$ and $k_{4}$.

Interestingly, the veering phenomenon was observed for variations in $k_{2}$ and $k_{\mathrm{t} 2}$ in the fourth and fifth modes [36]. Further, the veering regions are changed owing to the variations in $k_{2}$ and $k_{\mathrm{t} 2}$. Figure 7 shows the natural frequency loci of the fourth and fifth modes when $k_{2}$ and $k_{\mathrm{t} 2}$ vary from $1 / 1000$ to two times the baseline stiffness value. We illustrate the natural frequency loci using a three-dimensional graph, in order to more efficiently describe how different parameters cause modes to be influenced by the presence of the veering phenomenon associated with multi parameters $k_{2}$ and $k_{\mathrm{t} 2}$ [37]. Here, the upper and lower planes indicate the fourth and fifth natural frequencies, respectively. It can be seen that the two planes approach each other according to the variations in each parameter, and then they veer away abruptly. In the veering region, an interchange between the fourth and fifth eigenvectors also occurs and their mode shape subsequently changes. The presence of the eigenvalue veering phenomenon in the fourth and fifth modes is of prime importance because small variations in $k_{2}$ and $k_{\mathrm{t} 2}$ stiffness values can give rise to large changes in the eigenvectors within the veering region. In this human mathematical model, such veering phenomena play a key role in the design of seat and suspensions for a vehicle.

In order to investigate the veering quantitatively, a veering index value was calculated from the combination of the modal dependence factor (MDF) and the crosssensitivity quotient (CSQ) [38] as follows:

$$
\mathrm{VI}_{i j}=\mathrm{MDF}_{i j} \times \mathrm{CSQ}_{i j} \times \mathrm{MDF}_{j i} \text {. }
$$

The MDF was also defined as

$$
\mathrm{MDF}_{i j}=\frac{\left\{\left(\partial \phi_{i} / \partial a\right)^{\mathrm{T}} \mathbf{M} \phi_{j}\right\}^{2}}{\left(\partial \phi_{i} / \partial a\right)^{\mathrm{T}} \mathbf{M}\left(\partial \phi_{i} / \partial a\right)}
$$

where $\phi_{j}$ and $\phi_{j}$ are mass-normalized eigenvectors and $\mathbf{M}$ is the stiffness matrix. The eigenvector sensitivity with respect to parameter $a$ was approximated by

$$
\frac{\partial \phi_{i}}{\partial a} \approx-\frac{\phi_{i}^{T}(\partial \mathbf{M} / \partial \delta) \phi_{i}}{2}+\frac{\alpha_{i j}}{\Delta \lambda_{i j}} \phi_{i}
$$

where $\Delta \lambda_{i j}=\lambda_{j}-\lambda_{i}$, and $\lambda_{i}$ denotes the eigenvalue associated with $i$-th mode. The modal coupling $\alpha_{i j}$ was defined as

$$
\alpha_{i j}=\phi_{j}^{T}\left(\frac{\partial \mathbf{K}}{\partial a}-\lambda_{i} \frac{\partial \mathbf{M}}{\partial a}\right) \phi_{i}
$$

where $\mathbf{K}$ is the stiffness matrix. The CSQ is also given by 

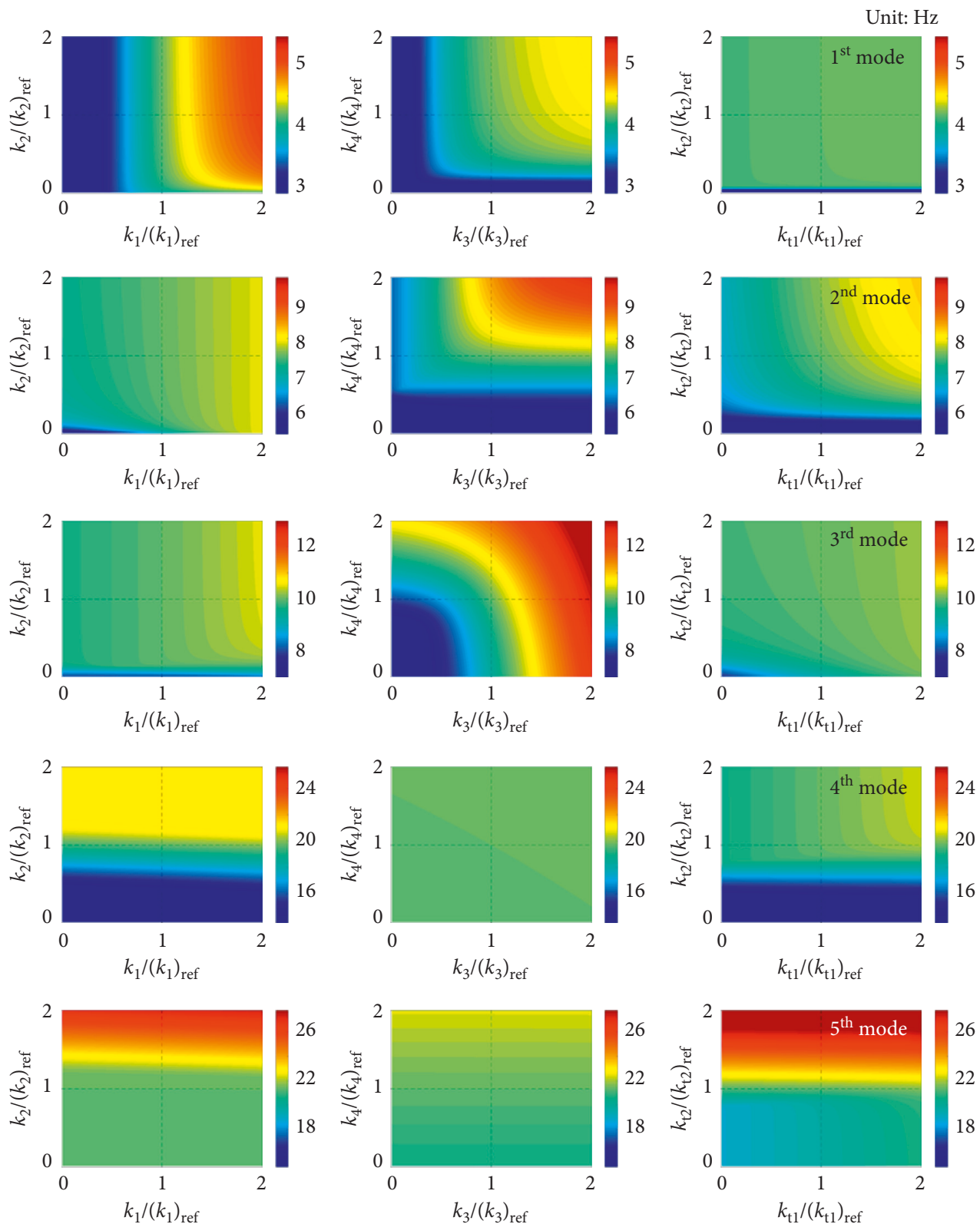

FIGURE 6: Natural frequency of the human model with respect to the translational and torsional stiffness values (dotted black vertical and horizontal line: baseline values of the stiffness; the top through the bottom rows, respectively, represent the first through the fifth modes).

$$
\mathrm{CSQ}_{i j}=\frac{\alpha_{i j}^{2}}{\alpha_{i j}^{2}+\left(\Delta \sigma_{j i} / 2\right)^{2}},
$$

where $\Delta \sigma_{j i}=\sigma_{j}-\sigma_{i}$, and $\sigma_{i}$ indicates the eigenvalue sensitivity. It can be written as

$$
\sigma_{i}=\frac{\partial \lambda_{i}}{\partial a}=\phi_{i}^{\mathrm{T}}\left(\frac{\partial \mathbf{M}}{\partial a}-\lambda_{i} \frac{\partial \mathbf{K}}{\partial a}\right) \phi_{i} .
$$

Figure 8 shows the veering indices with respect to $k_{2}$ and $k_{\mathrm{t} 2}$ stiffness. A veering index approaching 1 indicates that the effect of the veering phenomenon is relatively large. Thus, it can be expected that the folded area of the eigenvalue plane in Figure 7 represents the larger veering indices in Figure 8.
Further, the veering intensity band changes with respect to $k_{2}$ and $k_{\mathrm{t} 2}$ stiffness; this demonstrates that the eigenvalue curvature of the fourth and fifth modes is changed when stiffness values vary. Therefore, the natural frequency loci and veering index are considered simultaneously for better understanding of this veering phenomenon.

\section{Dynamic Simulation of Nonlinear Five-Degree-of-Freedom Human Model}

We carried out dynamic analysis of a five-degree-of-freedom nonlinear human model in the frequency domain to investigate its nonlinearity. Because nonlinear dynamic systems may have multiple responses at the same excitation 


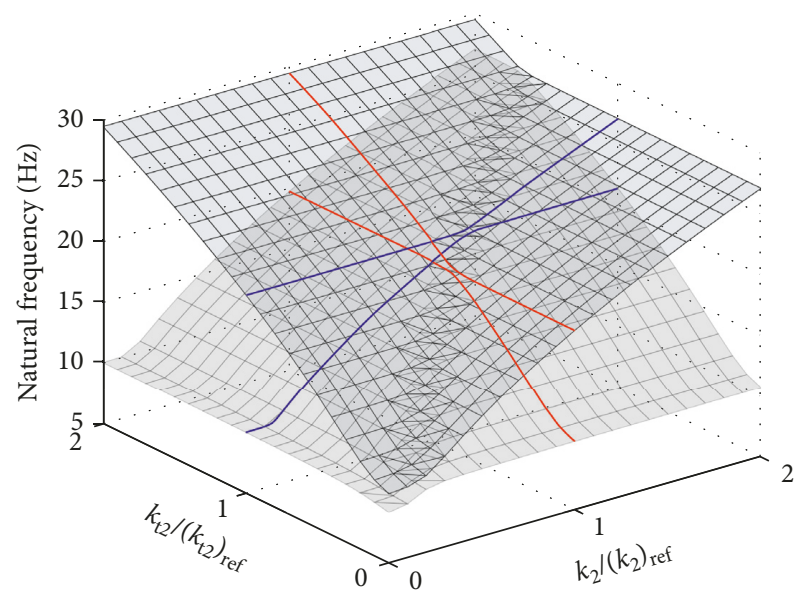

FIgURE 7: Mode veering between the fourth and fifth modes for variations in $k_{2}$ and $k_{\mathrm{t} 2}$, (lower plane: fourth natural frequencies; upper plane: fifth natural frequencies; red line: natural frequency loci when $k_{\mathrm{t} 2}$ varies with baseline value of $k_{2}$; blue line: natural frequency loci with respect to $k_{2}$ with baseline value of $k_{\mathrm{t} 2}$ ).

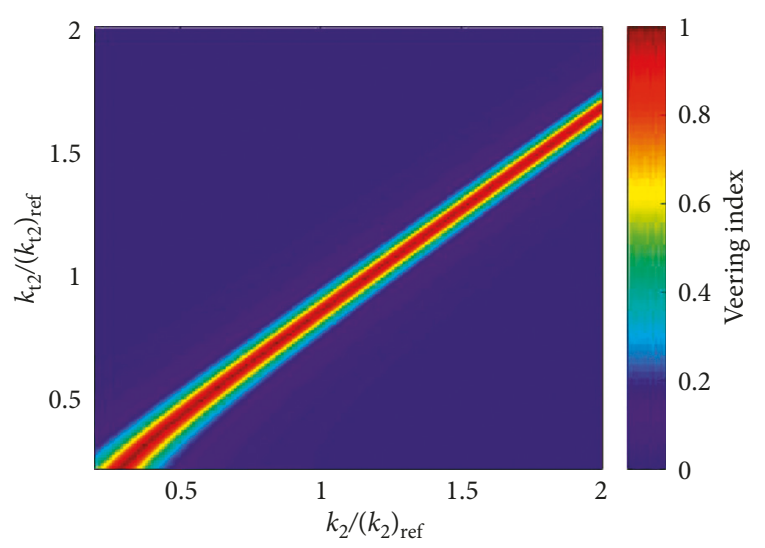

FIGURE 8: Veering index of the fourth and fifth modes with respect to $k_{2}$ and $k_{\mathrm{t} 2}$.

frequency, steady-state responses are needed to reach the solution for the frequency response function. We also used the optimized parameters of the human model in the analysis of the linear model. For the nonlinear human model, we applied a nonlinear force-displacement relationship to the translational springs to more accurately reflect the actual deformation behavior of seat foam and tissue. When the deflection of the translational spring is larger than the initial length of the corresponding spring, it means that the connection between the human body and the seat would be physically removed. Therefore, we considered that the tension force of the translational spring was set to zero when $\delta_{i} \geq \delta_{i 0}$. Here, the linear spring model was applied to $k_{3}$ and $k_{4}$ owing to horizontal constraints.

In order to compute the frequency response function of the five-degree-of-freedom nonlinear model near the fundamental frequency, the base floor was excited by harmonic excitation within a frequency range of $0.1 \mathrm{~Hz}$ to $5 \mathrm{~Hz}$ at intervals of $0.1 \mathrm{~Hz}$. To extract the steady-state responses of the human model, a sufficient excitation time must be taken into account to ensure steady-state responses; we set the simulation time at each frequency step to 50 seconds. The excitation frequency was also increased and then decreased uniformly using $0.1 \mathrm{~Hz}$ intervals. The displacement excitation was applied to the base of the nonlinear human model, and the amplitude of the excitation was changed in a range from $3 \mathrm{~mm}$ to $12 \mathrm{~mm}$ in $3 \mathrm{~mm}$ increments to analyze the dynamic characteristics of the human model with respect to the amplitude of the excitation displacement.

Figure 9 displays the translational and angular displacements with respect to the excitation frequency and amplitude in the frequency domain. The amplitudes of the translational and angular displacements increase as the excitation amplitude increases. It can be also confirmed that the first natural frequencies of each generalized coordinate have slightly different values. For example, the locations of the maximum amplitude for the vertical displacements of the hip joint, $z_{\mathrm{h}}$, and the angular displacements of the head, $\theta_{3}$, occur at $4.0 \mathrm{~Hz}$ and $4.3 \mathrm{~Hz}$, respectively. There is a minor difference between the first natural frequency of the nonlinear model and that of the linear model $\left(f_{1}\right.$ of linear model $=4.2 \mathrm{~Hz}$ ), and it may occur owing to its nonlinearity.

Importantly, it can be observed that a frequency softening phenomenon occurs in which the first natural frequency decreases with the increase in the excitation amplitude. The fundamental frequency of the vertical displacements of the hip joint and the angular displacements of the head was, respectively, reduced by approximately $15 \%$ and $14 \%$ while the excitation amplitude increased from $3 \mathrm{~mm}$ to $12 \mathrm{~mm}$. The first natural frequency exhibited a minor variation as the excitation amplitude increased from $3 \mathrm{~mm}$ to $6 \mathrm{~mm}$. The fundamental frequencies in the translational and angular displacements were rapidly decreased when the excitation amplitude exceeded $6 \mathrm{~mm}$. In addition, the jump phenomenon (in which the amplitude dramatically changes) becomes clear in the high excitation amplitude. As an example study, Mansfield et al. reported that the fundamental frequency of experimental subjects decreased by $22.2 \%$ from $5.4 \mathrm{~Hz}$ to $4.2 \mathrm{~Hz}$ based on median data and presumed that this frequency shifting would be caused by various complex causes such as muscle and tissue responses [11]. This phenomenon is more clearly observed in the frequency response functions of the rotational coordinates. In order to describe the multiple amplitudes at the same excitation frequency, the frequency response functions in the overlapped range are given using a zoom-in graph as shown in Figure 10; the amplitudes were calculated using $0.01 \mathrm{~Hz}$ intervals.

Figure 10 shows STH transmissibility data from the linear and nonlinear human models and the experiment. The results of the linear model and experimental data are the same as in Figure 4 and are replotted in Figure 10 for comparison with the frequency response of the nonlinear model. We also calculated the STH transmissibility of the nonlinear human model from the vertical displacements of the head at the center of gravity and provide the nonlinear human model results associated with an excitation amplitude of $3 \mathrm{~mm}$ for comparison with those from the linear model. Further, the STH transmissibility of the nonlinear 


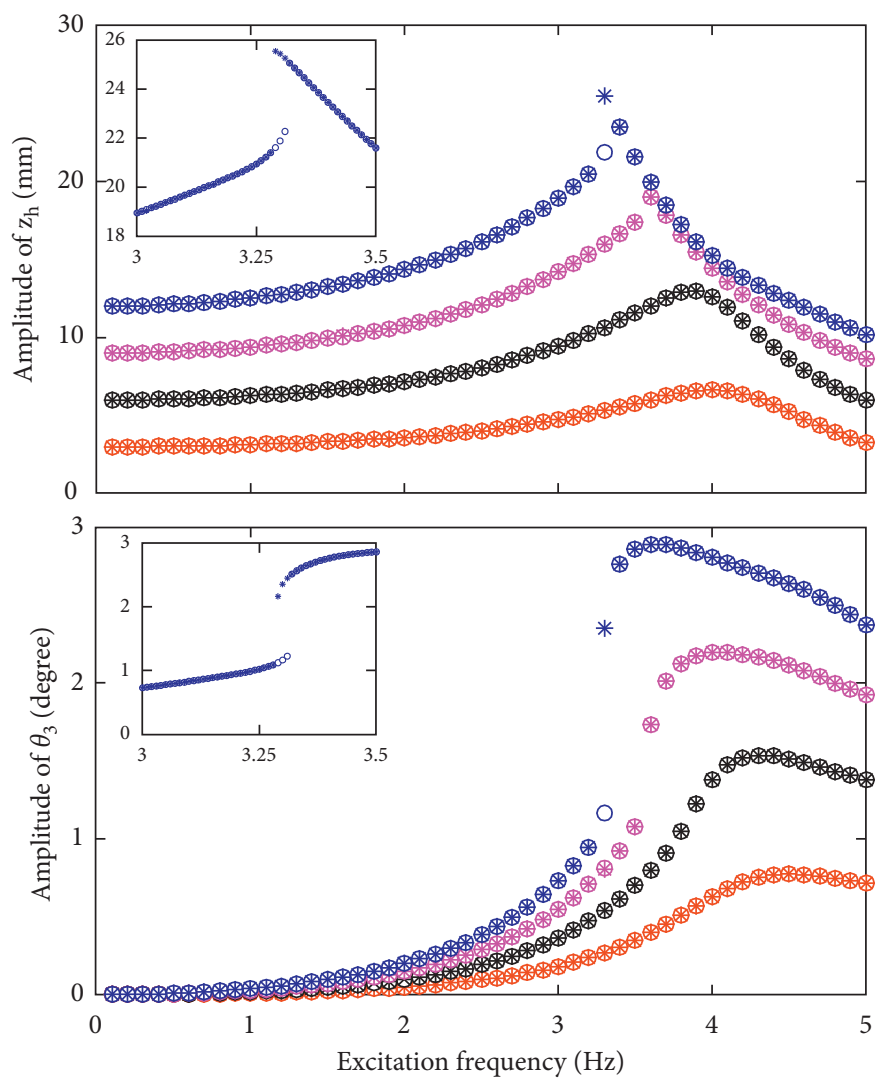

Figure 9: Amplitude of the vertical displacement of the hip joint and the angular displacement of the head with four different excitation amplitudes using the nonlinear human model (the circle and asterisk symbols indicate the amplitude values with increasing and decreasing excitation frequency, respectively).

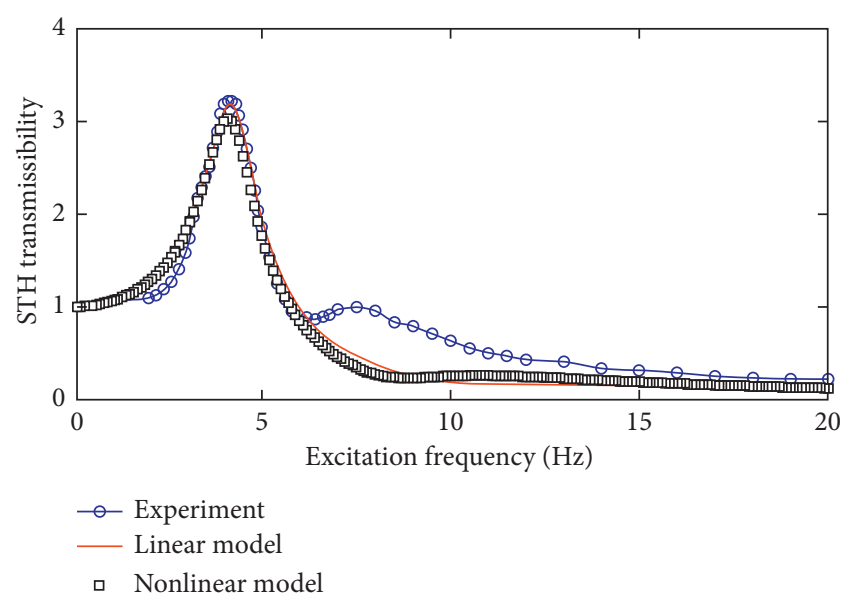

FIGURE 10: STH transmissibility of the linear and nonlinear proposed mathematical model and the experimental results [30] (nonlinear model: excitation amplitude of $3 \mathrm{~mm}$ ).

human model was calculated in a range of 0 to $20 \mathrm{~Hz}$ at frequency intervals of $0.1 \mathrm{~Hz}$.

It can be seen that the STH transmissibility of the nonlinear model is in good agreement with that of the linear model. The maximum amplitude of the nonlinear model is slightly lower than the amplitude of the experimental data and linear human model. This illustrates the fact that if the amplitude of the vertical excitation is relatively small, the linear human model could sufficiently represent frequency response function. However, it is more difficult to express the frequency response characteristics of the human body using the linear human model alone for large excitation amplitudes that cause shifting of the first natural frequency, and thus the necessity of the nonlinear model becomes more important. Further, the nonlinear model enables one to describe rapid changes in amplitude, such as those caused by the jump phenomenon. Therefore, it could be expected that the nonlinear model is more suitable for representing the vibrational characteristics of a seated human subjected to whole-body vibrations generated from the road, in which the profiles are changed from a smooth to a rough surface and vice versa.

\section{Conclusions}

In this study, we first derived the equations of motion for a human model. The determination of the human parameters was performed in three steps. The inertial and geometrical parameters were selected on the basis of anthropometry reference data comprised of measurable properties, and the stiffness and damping coefficients were extracted from the experimental STH results using the optimization process. The mode shapes were also obtained using the mass and stiffness matrix of the linearized model. 
In addition, we analyzed the variations in the natural frequencies of the linear human model when human parameters were varied. For this parametric study, the inclination angle and stiffness values were considered as the prominent parameters in the dynamic characteristics of the human model. As a result, the first natural frequency is most sensitive to the backrest angle, represented by spring stiffness value $k_{1}$. The variations in natural frequencies according to the change in stiffness values were compared with the configurations of the corresponding mode shape. Here, the mode veering occurs between the loci of the fourth and fifth natural frequencies for the variations in $k_{2}$ and $k_{\mathrm{t} 2}$.

The frequency response functions of the nonlinear human model were presented using the steady-state amplitude of translational and angular displacements caused by harmonic base excitation. We note that frequency shifting was observed in the first mode, and various studies have reported similar phenomenon. Thus, the proposed human model could be reasonably expected to accurately exhibit the dynamic response characteristics of a seated human.

\section{Appendix}

\section{Appendix A}

The elements of the matrix in Equation (5) are

$$
\begin{aligned}
& A_{11}=m_{1}+m_{2}+m_{3}, \\
& \mathrm{~A}_{13}=-\frac{1}{2} m_{1} L_{\mathrm{th}} \sin \theta_{1}, \\
& \mathrm{~A}_{14}=-\frac{1}{2} L_{\mathrm{tr}}\left(m_{2}+2 m_{3}\right) \sin \theta_{2}, \\
& \mathrm{~A}_{15}=-\frac{1}{2} m_{3} L_{\mathrm{h}} \ddot{\theta}_{3} \sin \theta_{3}, \\
& A_{22}=m_{1}+m_{2}+m_{3}, \\
& A_{23}=\frac{1}{2} m_{1} L_{\mathrm{th}} \cos \theta_{1}, \\
& A_{24}=\frac{1}{2} L_{\mathrm{tr}}\left(m_{2}+2 m_{3}\right) \cos \theta_{2}, \\
& A_{25}=\frac{1}{2} m_{3} L_{\mathrm{h}} \cos \theta_{3}, \\
& A_{33}=J_{1}+\frac{1}{4} m_{1} L_{\mathrm{th}}^{2}, \\
& A_{55}=J_{3}+\frac{1}{4} m_{3} L_{\mathrm{h}}^{2}, \\
& A_{45}=\frac{1}{2} m_{3} L_{\mathrm{tr}} L_{\mathrm{h}} \cos \left(\theta_{3}-\theta_{2}\right), \\
& \mathrm{tr}
\end{aligned}
$$

$$
\begin{aligned}
& B_{1}= \frac{1}{2} m_{1} L_{\mathrm{th}}\left(\dot{\theta}_{1}^{2} \cos \theta_{1}\right)+\frac{1}{2} L_{\mathrm{tr}}\left(m_{2}+2 m_{3}\right)\left(\dot{\theta}_{2}^{2} \cos \theta_{2}\right) \\
&+\frac{1}{2} m_{3} L_{\mathrm{h}}\left(\dot{\theta}_{3}^{2} \cos \theta_{3}\right)-\left(c_{3} \dot{\delta}_{3}+c_{4} \dot{\delta}_{4}+k_{3} \delta_{3}+k_{4} \delta_{4}\right) \\
& \cdot \sin \theta_{20}, \\
& B_{2}= \frac{1}{2} m_{1} L_{\mathrm{th}} \dot{\theta}_{1}^{2} \sin \theta_{1}+\frac{1}{2} L_{\mathrm{tr}}\left(m_{2}+2 m_{3}\right) \dot{\theta}_{2}^{2} \sin \theta_{2} \\
&+\frac{1}{2} m_{3} L_{\mathrm{h}} \dot{\theta}_{3}^{2} \sin \theta_{3}-c_{1} \dot{\delta}_{1}-c_{2} \dot{\delta}_{2}-k_{1} \delta_{1}-k_{2} \delta_{2} \\
&+\left(c_{3} \dot{\delta}_{3}+c_{4} \dot{\delta}_{4}+k_{3} \delta_{3}+k_{4} \delta_{4}\right) \cos \theta_{20}-\left(m_{1}+m_{2}+m_{3}\right) g \\
& B_{3}=-\left(c_{1} \dot{\delta}_{1}+k_{1} \delta_{1}\right)\left(\frac{1}{2} L_{\mathrm{t} 1} \sin \theta_{1}+L_{1} \cos \theta_{1}\right) \\
&-\left(c_{2} \dot{\delta}_{2}+k_{2} \delta_{2}\right)\left(\frac{1}{2} L_{\mathrm{t} 1} \sin \theta_{1}+L_{2} \cos \theta_{1}\right)+c_{\mathrm{t} 1} \dot{\delta}_{\mathrm{t} 1} \\
&+k_{\mathrm{t} 1} \delta_{\mathrm{t} 1}-\frac{1}{2} m_{1} g L_{\mathrm{th}} \cos \theta_{1}, \\
&-\frac{1}{2} m_{3} g L_{\mathrm{h}} \cos \theta_{3}, \\
& B_{5}=-\frac{1}{2} m_{3} L_{\mathrm{tr}} L_{\mathrm{h}} \dot{\theta}_{2}^{2} \sin \left(\theta_{3}-\theta_{2}\right)-c_{\mathrm{t} 2} \dot{\delta}_{\mathrm{t} 2}-k_{\mathrm{t} 2} \delta_{\mathrm{t} 2} \\
&+m_{3} L_{\mathrm{tr}} L_{\mathrm{h}} \dot{\theta}_{3}^{2} \sin \left(\theta_{3}-\theta_{2}\right)-\left(c_{3} \dot{\delta}_{3}+k_{3} \delta_{3}\right) \\
& \cdot\left\{\frac{1}{2} L_{\mathrm{t} 2} \sin \left(\theta_{2}-\theta_{20}\right)-L_{3} \cos \left(\theta_{2}-\theta_{20}\right)\right\}-\left(c_{4} \dot{\delta}_{4}+k_{4} \delta_{4}\right) \\
& \cdot\left\{\frac{1}{2} L_{\mathrm{t} 2} \sin \left(\theta_{2}-\theta_{20}\right)-L_{4} \cos \left(\theta_{2}-\theta_{20}\right)\right\}-c_{\mathrm{t} 1} \dot{\delta}_{\mathrm{t} 1} \\
& B_{4} k_{\mathrm{t} 2} \delta_{\mathrm{t} 2}-\left(\frac{1}{2} m_{2}+m_{3}\right) g L_{\mathrm{tr}} \cos \theta_{2}, \\
& \\
&
\end{aligned}
$$

where $L_{i}$ is the distance from the hip joint to the position where the $i$-th translational spring and damper are connected. $L_{\mathrm{t} 1}$ and $L_{\mathrm{t} 2}$ denote the thickness values of the lower body and trunk, respectively. The subscript 0 of $\theta_{i 0}$ indicates the initial inclination angle. Moreover, the translational and torsional spring displacements are given below.

$$
\begin{aligned}
\delta_{1}= & z_{\mathrm{h}}-\frac{L_{\mathrm{t} 1}}{2} \cos \theta_{1}+L_{1} \sin \theta_{1}-z_{\mathrm{b}}-\delta_{10}, \\
\delta_{2}= & z_{\mathrm{h}}-\frac{L_{\mathrm{t} 1}}{2} \cos \theta_{1}+L_{2} \sin \theta_{1}-z_{\mathrm{b}}-\delta_{20}, \\
\delta_{3}= & x_{\mathrm{h}} \sin \theta_{20}-z_{\mathrm{h}} \cos \theta_{20}-L_{3} \sin \left(\theta_{2}-\theta_{20}\right) \\
& -\frac{L_{\mathrm{t} 2}}{2} \cos \left(\theta_{2}-\theta_{20}\right)-x_{\mathrm{s} 3} \sin \theta_{20}+z_{\mathrm{s} 3} \cos \theta_{20}-\delta_{30}, \\
\delta_{4}= & x_{\mathrm{h}} \sin \theta_{20}-z_{\mathrm{h}} \cos \theta_{20}-L_{4} \sin \left(\theta_{2}-\theta_{20}\right) \\
& -\frac{L_{\mathrm{t} 2}}{2} \cos \left(\theta_{2}-\theta_{20}\right)-x_{\mathrm{s} 4} \sin \theta_{20}+z_{\mathrm{s} 4} \cos \theta_{20}-\delta_{40}, \\
\delta_{\mathrm{t} 1}= & \theta_{2}-\theta_{1}-\delta_{t 10}, \\
\delta_{\mathrm{t} 2}= & \theta_{3}-\theta_{2}-\delta_{t 20},
\end{aligned}
$$


where $x_{\mathrm{s} 3}, z_{\mathrm{s} 3}, x_{\mathrm{s} 4}$, and $z_{\mathrm{s} 4}$ are the connected locations between the fixed backrest of the seat and the trunk of the human mathematical model. $\delta_{i 0}$ and $\delta_{t i 0}$ denote the initial length of the translational springs and the initial angle of the torsional springs, respectively.

\section{Appendix B}

The elements of the mass, damping, and stiffness matrix of the five-degree-of-freedom linear model are given by

$$
\begin{aligned}
& M_{11}=m_{1}+m_{2}+m_{3} \text {, } \\
& M_{13}=-\frac{1}{2} m_{1} L_{\text {th }} \sin \theta_{10} \text {, } \\
& M_{14}=-\frac{1}{2}\left(m_{2}+2 m_{3}\right) L_{\mathrm{tr}} \sin \theta_{20}, \\
& M_{15}=-\frac{1}{2} m_{3} L_{\mathrm{h}} \sin \theta_{30} \text {, } \\
& M_{22}=m_{1}+m_{2}+m_{3} \\
& M_{23}=\frac{1}{2} m_{1} L_{\mathrm{th}} \cos \theta_{10} \text {, } \\
& M_{24}=\frac{1}{2}\left(m_{2}+2 m_{3}\right) L_{\mathrm{tr}} \cos \theta_{20}, \\
& M_{25}=\frac{1}{2} m_{3} L_{\mathrm{h}} \cos \theta_{30} \text {, } \\
& M_{33}=J_{1}+\frac{1}{4} m_{1} L_{\mathrm{th}}^{2}, \\
& M_{44}=J_{2}+\frac{1}{4} m_{2} L_{\mathrm{tr}}^{2}+m_{3} L_{\mathrm{tr}}^{2}, \\
& M_{45}=\frac{1}{2} m_{3} L_{\mathrm{h}} L_{\text {tr }} \cos \left(\theta_{20}-\theta_{30}\right) \text {, } \\
& M_{55}=J_{3}+\frac{1}{4} m_{3} L_{\mathrm{h}}^{2}, \\
& C_{11}=\left(c_{3}+c_{4}\right) \sin ^{2} \theta_{20}, C_{12}=-\left(c_{3}+c_{4}\right) \sin \theta_{20} \cos \theta_{20}, \\
& C_{14}=-\left(c_{3} L_{3}+c_{4} L_{3}\right) \sin \theta_{20} \text {, } \\
& C_{22}=c_{1}+c_{2}+\left(c_{3}+c_{4}\right) \cos ^{2} \theta_{20} \text {, } \\
& C_{23}=\frac{1}{2}\left\{2\left(c_{1} L_{1}+c_{2} L_{2}\right) \cos \theta_{10}+\left(c_{1}+c_{2}\right) L_{\mathrm{t} 1} \sin \theta_{10}\right\}, \\
& C_{24}=\left(c_{3} L_{3}+c_{4} L_{3}\right) \cos \theta_{20} \text {, } \\
& C_{33}=\left(c_{1} L_{1}^{2}+c_{2} L_{2}^{2}\right) \cos ^{2} \theta_{10}+\left(c_{1} L_{1}+c_{2} L_{2}\right) L_{\mathrm{t} 1} \sin \theta_{10} \cos \theta_{10} \\
& +\frac{1}{4}\left\{4 c_{\mathrm{t} 1}+\left(c_{1}+c_{2}\right) L_{\mathrm{t} 1}^{2} \sin ^{2} \theta_{10}\right\}, \\
& C_{34}=-c_{\mathrm{t} 1} \text {, } \\
& C_{44}=c_{\mathrm{t} 1}+c_{\mathrm{t} 2}+c_{3} L_{3}^{2}+c_{4} L_{4}^{2} \text {, } \\
& C_{45}=-c_{\mathrm{t} 2} \text {, } \\
& C_{55}=c_{\mathrm{t} 2} \text {, } \\
& K_{11}=\left(k_{3}+k_{4}\right) \sin ^{2} \theta_{20} \text {, }
\end{aligned}
$$

$$
\begin{aligned}
K_{12}= & -\left(k_{3}+k_{4}\right) \sin \theta_{20} \cos \theta_{20}, \\
K_{14}= & -\left(k_{3} L_{3}+k_{4} L_{3}\right) \sin \theta_{20}, \\
K_{22}= & k_{1}+k_{2}+\left(k_{3}+k_{4}\right) \cos ^{2} \theta_{20}, \\
K_{23}= & \frac{1}{2}\left\{2\left(k_{1} L_{1}+k_{2} L_{2}\right) \cos \theta_{10}+\left(k_{1}+k_{2}\right) L_{\mathrm{t} 1} \sin \theta_{10}\right\}, \\
K_{24}= & \left(k_{3} L_{3}+k_{4} L_{3}\right) \cos \theta_{20}, \\
K_{33}= & \left(k_{1} L_{1}^{2}+k_{2} L_{2}^{2}\right) \cos ^{2} \theta_{10}+\left(k_{1} L_{1}+k_{2} L_{2}\right) L_{\mathrm{t} 1} \sin \theta_{10} \cos \theta_{10} \\
& +\frac{1}{4}\left\{4 k_{\mathrm{t} 1}-2 m_{1} g L_{\mathrm{th}} \sin \theta_{10}+\left(k_{1}+k_{2}\right) L_{\mathrm{t} 1}^{2} \sin \theta_{10}\right\}, \\
K_{34}= & -k_{\mathrm{t} 1}, \\
K_{44}= & k_{\mathrm{t} 1}+k_{\mathrm{t} 2}+k_{3} L_{3}^{2}+k_{4} L_{4}^{2}-\frac{1}{2}\left(m_{2}+2 m_{3}\right) g L_{\mathrm{tr}} \sin \theta_{20}, \\
K_{45}= & -k_{\mathrm{t} 2}, \\
K_{55}= & k_{\mathrm{t} 2}-\frac{1}{2} m_{3} g L_{\mathrm{h}} \sin \theta_{30}, \\
C_{b 2}= & -\left(c_{1}+c_{2}\right), \\
C_{b 3}= & -\frac{1}{2}\left\{2\left(c_{1} L_{1}+c_{2} L_{2}\right) \cos \theta_{10}+\left(c_{1}+c_{2}\right) L_{\mathrm{t} 1} \sin \theta_{10}\right\}, \\
K_{b 2}= & -\left(k_{1}+k_{2}\right), \\
K_{b 3}= & -\frac{1}{2}\left\{2\left(k_{1} L_{1}+k_{2} L_{2}\right) \cos \theta_{10}+\left(k_{1}+k_{2}\right) L_{\mathrm{t} 1} \sin \theta_{10}\right\} . \\
&
\end{aligned}
$$

\section{Data Availability}

The length and thickness data of the Korean male are accessible at https://sizekorea.kr/in the section of $5^{\text {th }}$ measurement survey.

\section{Conflicts of Interest}

The authors declare that they have no conflicts of interest.

\section{Acknowledgments}

This work was supported by the National Research Foundation of Korea (NRF) grant funded by the Korea government (Ministry of Education) (No. 2015R1D1A 1A01060582).

\section{References}

[1] T. E. Fairley and M. J. Griffin, "The apparent mass of the seated human body: vertical vibration," Journal of Biomechanics, vol. 22, no. 2, pp. 81-94, 1989.

[2] Z. Zhou and M. J. Griffin, "Response of the seated human body to whole-body vertical vibration: biodynamic responses to sinusoidal and random vibration," Ergonomics, vol. 57, no. 5, pp. 693-713, 2014.

[3] E. Kim, M. Fard, and K. Kato, "Characterisation of the human-seat coupling in response to vibration," Ergonomics, vol. 60, no. 8, pp. 1085-1100, 2017. 
[4] M. Tarabini, S. Solbiati, G. Moschioni, B. Saggin, and D. Scaccabarozzi, "Analysis of non-linear response of the human body to vertical whole-body vibration," Ergonomics, vol. 57, no. 11, pp. 1711-1723, 2014.

[5] S. D. Smith, J. A. Smith, and D. R. Bowden, "Transmission characteristics of suspension seats in multi-axis vibration environments," International Journal of Industrial Ergonomics, vol. 38, no. 5-6, pp. 434-446, 2008.

[6] S. Rakheja, I. Stiharu, H. Zhang, and P. É. Boileau, "Seated occupant interactions with seat backrest and pan, and biodynamic responses under vertical vibration," Journal of Sound and Vibration, vol. 298, no. 3, pp. 651-671, 2006.

[7] J. L. Coyte, D. Stirling, H. Du, and M. Ros, "Seated wholebody vibration analysis, technologies, and modeling: a survey," IEEE Transactions on Systems, Man, and Cybernetics: Systems, vol. 46, no. 6, pp. 725-739, 2016.

[8] W. Wang, S. Rakheja, and P. É. Boileau, "Effects of sitting postures on biodynamic response of seated occupants under vertical vibration," International Journal of Industrial Ergonomics, vol. 34, no. 4, pp. 289-306, 2004.

[9] I. Hermanns, N. Raffler, R. P. Ellegast, S. Fischer, and B. Göres, "Simultaneous field measuring method of vibration and body posture for assessment of seated occupational driving tasks," International Journal of Industrial Ergonomics, vol. 38, no. 3, pp. 255-263, 2008.

[10] S. Rahmatalla and J. DeShaw, "Predictive discomfort of nonneutral head-neck postures in fore-aft whole-body vibration," Ergonomics, vol. 54, no. 3, pp. 263-272, 2011.

[11] N. J. Mansfield and M. J. Griffin, "Non-linearities in apparent mass and transmissibility during exposure to whole-body vertical vibration," Journal of Biomechanics, vol. 33, no. 8, pp. 933-941, 2000.

[12] Y. Qiu and M. J. Griffin, "Transmission of fore-aft vibration to a car seat using field tests and laboratory simulation," Journal of Sound and Vibration, vol. 264, no. 1, pp. 135-155, 2003.

[13] S. Tufano and M. J. Griffin, "Nonlinearity in the vertical transmissibility of seating: the role of the human body apparent mass and seat dynamic stiffness," Vehicle System Dynamics, vol. 51, no. 1, pp. 122-138, 2013.

[14] N. A. Vavalle, D. P. Moreno, A. C. Rhyne, J. D. Stitzel, and F. S. Gayzik, "Lateral impact validation of a geometrically accurate full body finite element model for blunt injury prediction," Annals of Biomedical Engineering, vol. 41, no. 3, pp. 497-512, 2013.

[15] A. Siefert, S. Pankoke, and H. P. Wölfel, "Virtual optimisation of car passenger seats: simulation of static and dynamic effects on drivers' seating comfort," International Journal of Industrial Ergonomics, vol. 38, no. 5, pp. 410-424, 2008.

[16] K. Butz, C. Spurlock, R. Roy et al., "Development of the CAVEMAN human body model: validation of lower extremity sub-injurious response to vertical accelerative loading," Stapp Car Crash Journal, vol. 61, pp. 175-209, 2017.

[17] L. Wei and J. Griffin, "The prediction of seat transmissibility from measures of seat impedance," Journal of Sound and Vibration, vol. 214, no. 1, pp. 121-137, 1998.

[18] S. B. Choi and Y. M. Han, "Vibration control of electrorheological seat suspension with human-body model using sliding mode control," Journal of Sound and Vibration, vol. 303, no. 1-2, pp. 391-404, 2007.

[19] X. X. Bai, S. X. Xu, W. Cheng, and L. J. Qian, "On 4-degree-offreedom biodynamic models of seated occupants: lumpedparameter modeling," Journal of Sound and Vibration, vol. 402, pp. 122-141, 2017.
[20] Y. Matsumoto and M. J. Griffin, "Modelling the dynamic mechanisms associated with the principal resonance of the seated human body," Clinical Biomechanics, vol. 16, pp. S31-S44, 2001.

[21] Y. Cho and Y.S. Yoon, "Biomechanical model of human on seat with backrest for evaluating ride quality," International Journal of Industrial Ergonomics, vol. 27, no. 5, pp. 331-345, 2001.

[22] S. K. Kim, S. W. White, A. K. Bajaj, and P. Davies, "Simplified models of the vibration of mannequins in car seats," Journal of Sound and Vibration, vol. 264, no. 1, pp. 49-90, 2003.

[23] G. Zheng, Y. Qiu, and M. J. Griffin, "An analytic model of the in-line and cross-axis apparent mass of the seated human body exposed to vertical vibration with and without a backrest," Journal of Sound and Vibration, vol. 330, no. 26, pp. 6509-6525, 2011.

[24] N. Mohajer, H. Abdi, S. Nahavandi, and K. Nelson, "Directional and sectional ride comfort estimation using an integrated human biomechanical-seat foam model," Journal of Sound and Vibration, vol. 403, pp. 38-58, 2017.

[25] BS 6841, Measurement and Evaluation of Human Exposure to Whole-Body Mechanical Vibration and Repeated Shock, British Standard, London, 1987.

[26] M. J. Griffin, Handbook of Human Vibration, Academic Press, Cambridge, MA, USA, 1990.

[27] R. K. Ippili, P. Davies, A. K. Bajaj, and L. Hagenmeyer, "Nonlinear multi-body dynamic modeling of seat-occupant system with polyurethane seat and H-point prediction," International Journal of Industrial Ergonomics, vol. 38, no. 5, pp. 368-383, 2008.

[28] G. Joshi, A. K. Bajaj, and P. Davies, "Whole-body vibratory response study using a nonlinear multi-body model of seatoccupant system with viscoelastic flexible polyurethane foam," Industrial Health, vol. 48, no. 5, pp. 663-674, 2010.

[29] M. L. Ju, H. Jmal, R. Dupuis, and E. Aubry, "Visco-hyperelastic constitutive model for modeling the quasi-static behavior of polyurethane foam in large deformation," Polymer Engineering and Science, vol. 55, no. 8, pp. 1795-1804, 2015.

[30] C. C. Liang and C. F. Chiang, "Modeling of a seated human body exposed to vertical vibrations in various automotive postures," Industrial Health, vol. 46, no. 2, pp. 125-137, 2008.

[31] S. J. Park, S. C. Park, J. H. Kim, and C. B. Kim, "Biomechanical parameters on body segments of Korean adults," International Journal of Industrial Ergonomics, vol. 23, no. 1, pp. 23-31, 1999.

[32] Online database of the size of Korean body, http://sizekorea. $\mathrm{kr} /$.

[33] M. M. Verver, R. De Lange, J. F. A. M. van Hoof, and J. S. Wismans, "Aspects of seat modelling for seating comfort analysis," Applied Ergonomics, vol. 36, no. 1, pp. 33-42, 2005.

[34] D. M. Brienza, P. E. Karg, and C. E. Brubaker, "Seat cushion design for elderly wheelchair users based on minimization of soft tissue deformation using stiffness and pressure measurements," IEEE Transactions on Rehabilitation Engineering, vol. 4, no. 2, pp. 320-327, 1996.

[35] M. G. Toward and M. J. Griffin, "Apparent mass of the human body in the vertical direction: effect of seat backrest," Journal of Sound and Vibration, vol. 327, no. 3, pp. 657-669, 2009.

[36] A. W. Leissa, "On a curve veering aberration," Zeitschrift für Angewandte Mathematik und Physik ZAMP, vol. 25, no. 1, pp. 99-111, 1974.

[37] A. Gallina, L. Pichler, and T. Uhl, "Enhanced meta-modelling technique for analysis of mode crossing, mode veering and mode coalescence in structural dynamics," Mechanical Systems and Signal Processing, vol. 26, no. 7, pp. 2297-2312, 2011. 
[38] J. L. du Bois, S. Adhikari, and N. A. Lieven, "On the quantification of eigenvalue curve veering: a veering index," Journal of Applied Mechanics, vol. 78, no. 4, article 041007, 2011. 


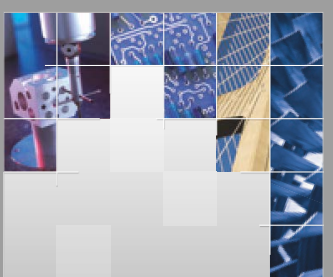

\section{Enfincering}
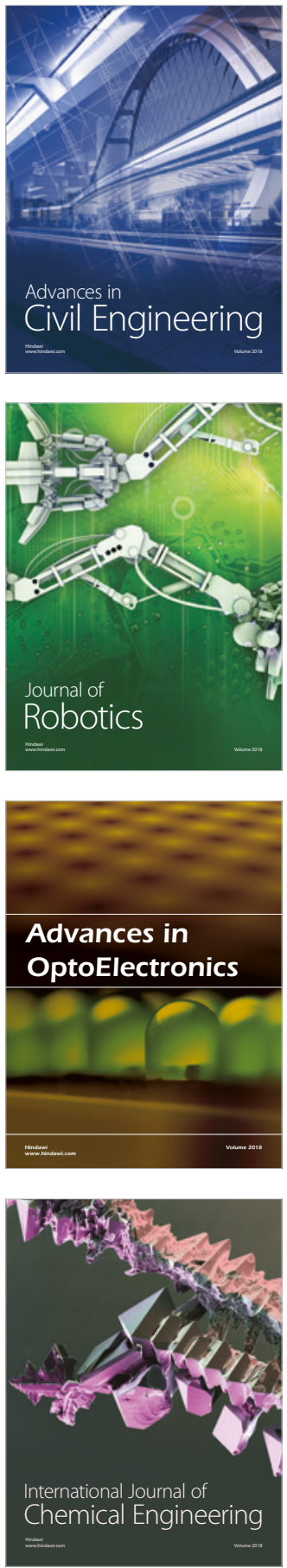

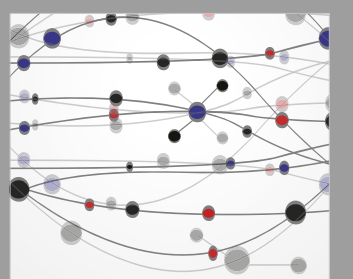

\section{Rotating \\ Machinery}

The Scientific World Journal

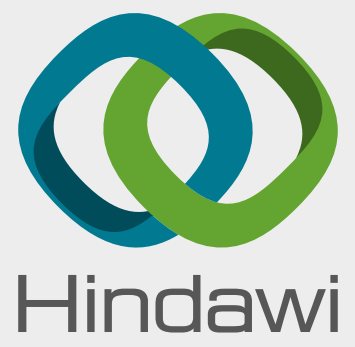

Submit your manuscripts at

www.hindawi.com
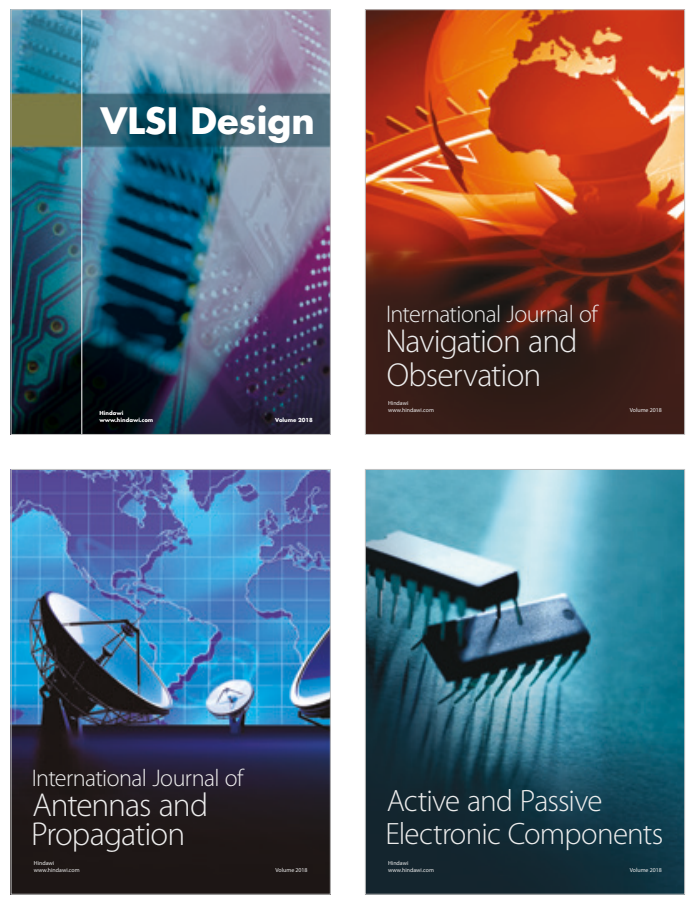
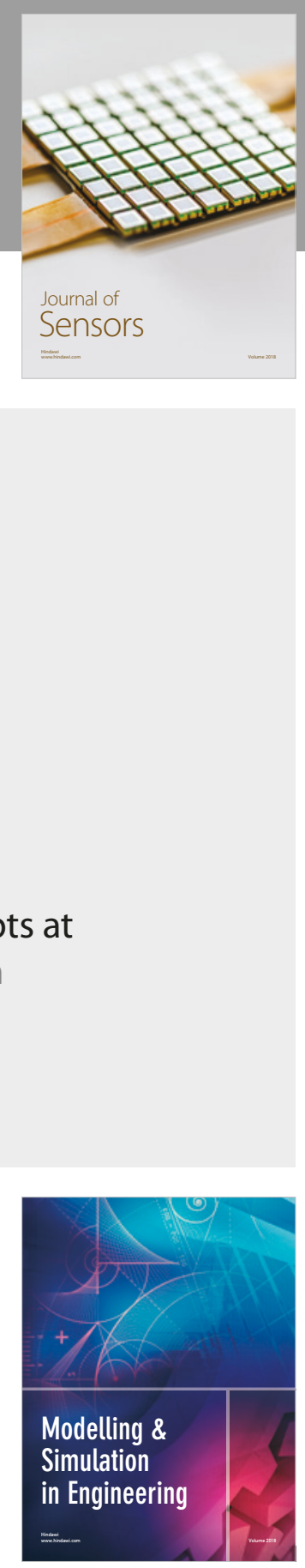

\section{Advances \\ Multimedia}
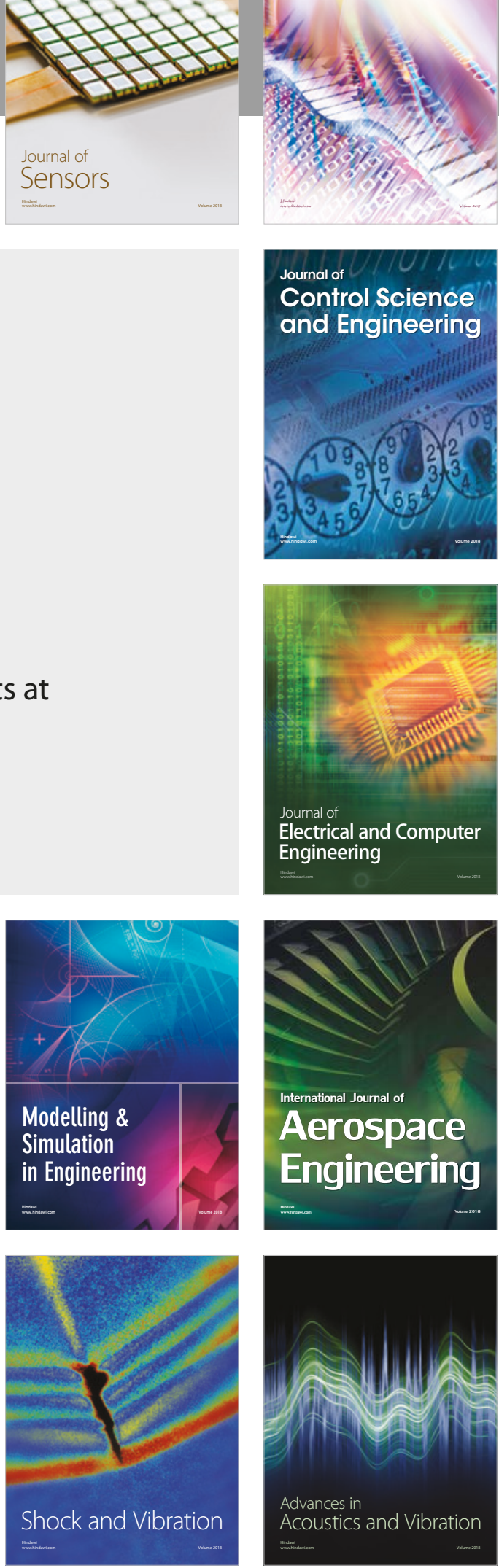